\title{
Research on Transmission Network Planning Model based on LCC Theory and Improved AFSA
}

\section{Fuyu HUA}

School of Economics and Management, North China Electric Power University, Beijing, China

\section{Jinhua HUANG}

State Grid Zhejiang Electric Power Company Economic Research Institute, Hangzhou, China

Dongxiao NIU \& Yali HUANG

School of Economics and Management, North China Electric Power University, Beijing, China

\begin{abstract}
The cost of power project can be controlled in the preliminary design stage, which means reducing life-cycle costs of electric power project in network planning stage. According to life-cycle cost theory, the costs of network project were analyzed and the full life-cycle cost model was established. Based on the improved Artificial Fish Swarm Algorithm, the problem was solved and the lowest life cycle cost of the grid plan was found. This paper not only verified the applicability of full life-cycle costs theory in network planning, but also contributed to reduce the full life-cycle costs of power project and improve the economics of grid construction.
\end{abstract}

KEYWORD: Artificial Fish Swarm Algorithm (AFSA); network planning; life cycle cost; objective optimization

\section{INTRODUCTION}

In recent years, electricity demand increased greatly and the electric power industry investment enlarged continuously. Coupled with the emergence of new technologies, new methods, the construction cost of power engineering project becomes higher and higher. Considering the whole life cycle cost of power project and optimizing it can significantly reduce the power project construction cost, so as to improve the economy of the project [1]. Besides, the rapid development of artificial intelligence algorithm provides a new method to solve the problem of transmission network planning. Among them, Artificial Fish Swarm Algorithm (AFSA) makes the artificial fish have the memory ability in the exploring process by introducing tabu search algorithm and improving part of artificial fish behavior, which can ensure efficiency and diversity of searching and obtain the global optimum rather than the local optimum.

Based on life cycle cost management theory, this paper analyzed the cost of power project and built the life cycle cost model first. Then the improved Artificial Fish Swarm Algorithm is used to solve the model to get the optimized grid plan, so as to control the project cost and improve the power project economic effectiveness [2].

\section{AFSA AND IMPROVED AFSA}

The basic process of artificial fish swarm algorithm (AFSA) is shown in Figure 1:

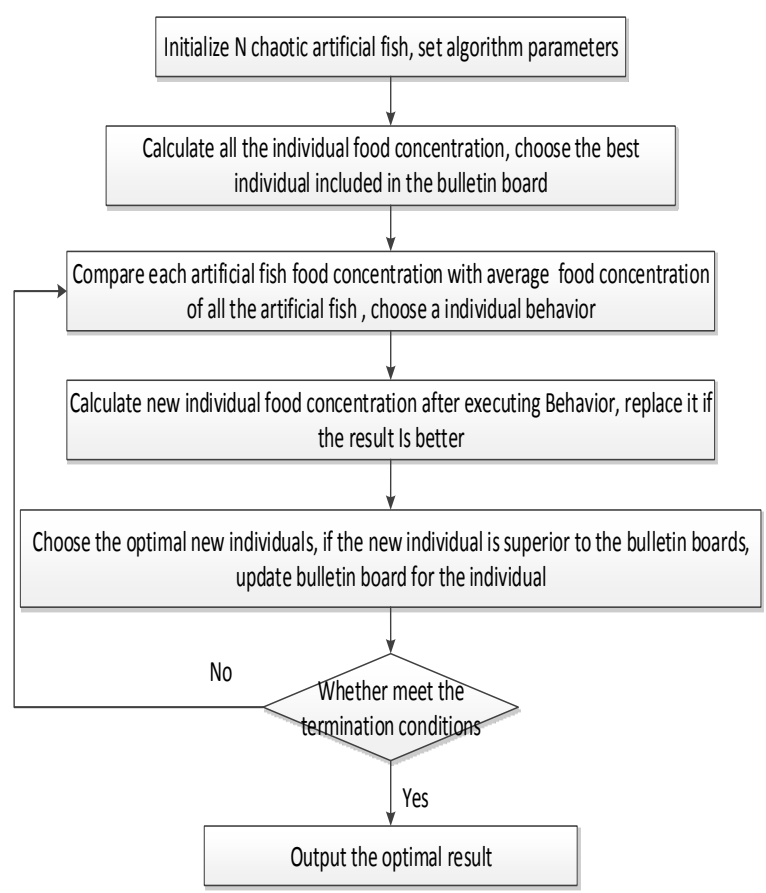

Fig.1 AFSA Flow chart

As a kind of random search algorithm, AFSA has the advantages of low requirement for the initial value, good global convergence, strong robustness, easy realization. However, it has some defects such 
as low optimization accuracy, slow convergence speed later, which are caused by the uncertainty of vision and step.

Aimed at the shortage of AFSA, this paper adopts adaptive vision and step to improve the basic algorithm to enhance the optimization performance. On the basis of analyzing the defects of AFSA, the algorithm is optimized by introducing apparent step coefficient. Specific as follows: in the process of iteration, determine an apparent step coefficient $\alpha \in[0,1]$, introduce the function

$$
\text { Step }=\alpha \times \text { Visual }
$$

as the maximum step size of artificial fish, thereby combine the vision and step length, which effectively reduce the initial variable values, so as to improve the operation speed and precision of the algorithm to some extent. To be sure, vision and step only change adaptively in foraging behavior. They stay the same in crash behavior and cluster behavior.

\section{THE OPTIMIZATION MODEL OF TRANSMISSION NETWORK ECONOMIC PLANNING BASED ON AFSA}

\subsection{Model construction}

Through the above analysis and basic contents and characteristics of LCC management, the life cycle cost of transmission network project is selected as the main planning object in this paper. Thus the optimization model can be established for seeking to planning schemes which are optimal reliable and economic and satisfy the technical constraints. Without considering the substation's location and capacity planning, this network planning model only considers the optimization problem of transmission lines as the research object. Suppose the locations, supply and load distribution of each substation are known in advance, according to the existing network structure and parameters, a reasonable choice of candidate transmission lines in the network can be made based on the model. With minimum present value of Life Cycle Cost of the network planning as the objective function, the mathematical expression of the model is shown as follows:

$$
\begin{aligned}
& \min L C C=\sum_{k=1}^{N}\left(\sum _ { u ( k ) } \left(\sum_{i \in f(k)} C_{l k, i} \times P_{1}+\right.\right. \\
& \left.\left.\left(C_{O k}+C_{M k}+C_{F k}\right) \times P V_{s u m}+\sum_{l \in p(k)} C_{D k, l} \times P_{2}+M_{l} L\right)\right) \\
& \text { s.t }\left\{\begin{array}{l}
A P=D \\
P_{i}<P_{i \max } \\
\text { radial network }
\end{array}\right.
\end{aligned}
$$

The parameters in the model respectively represents:
$L C C \_$_

$N \_$number of planning period;

$r-$ discount rate;

$u(k)$ — transmission line set in period $\mathrm{k}$;

$f(k) \longrightarrow$ newly built transmission line set in period $\mathrm{k}$;

$\mathrm{k}$;

$p(k) —$ removed transmission line set in period

$M_{l} \longrightarrow$ punishment coefficient of network overload;

$L-$ value of network Overload ;

$A \longrightarrow$ node incidence matrix;

$P$ — capacity of transmission lines;

$D \longrightarrow$ load demand;

$P_{i} \longrightarrow$ capacity of transmission line I;

$P_{i \max } \longrightarrow$ maximum capacity of transmission line $\mathrm{i}$;

$$
P_{1}=\frac{1}{(1+r)^{m(k-1)}}, \quad P_{2}=\frac{1}{(1+r)^{m(k)}}, P V_{\text {sum }}=\frac{(1+r)^{g(i)}-1}{r(1+r)^{m(k-1)+g(i)}}
$$

\subsection{Model solution method-Artificial Fish Swarm Algorithm (AFSA)}

\subsubsection{Algorithm realization}

\subsubsection{Code of artificial fish}

Firstly, all candidate transmission lines are supposed to be sequenced according to the node number on both ends of each line, which determines the coordinates of the artificial fish position. Specifically, when one bit of the coordinate is 1 , the corresponding line is supposed to be newly built in the network; when it comes to 0 , the corresponding line is not supposed to be selected in the network. Therefore, the number of coordinates of all artificial fish position equals to the number of candidate transmission lines, while each artificial fish position represents a network planning scheme. For example, suppose there are 7 candidate lines in the network and the position of one artificial fish is coded as [0011010], then it means the candidate transmission line 3, 4 and 6 are selected to be built in the planning scheme while line1, 2, 5, and 7 not involved the scheme.

\subsubsection{Initialization of artificial fish}

The second step is randomly generating a certain amount of artificial fish, meaning position coordinate of each fish is randomly assigned with 0 or 1. In this way the artificial fish can be initialized, but the food concentration (the minimum target LCC) of the initialized position is generally not high.

\subsubsection{Behavior of artificial fish}

After the first and second step, the initialized artificial fish will begin to conduct prey or follow behavior. In the prey behavior, the present position 
$X_{i}$ of the artificial fish can be changed to $X_{j}$ by random inverting operation according to the value of visual. For instance, suppose $X_{i}=[1010011]$ and visual $=3$, then the new position $X_{j}$ of the artificial fish can probably be [1001010], [1000101], [0001010] and so on. In the follow behavior, still assuming the present position and food concentration of the artificial fish is respectively $X_{i}$ and $Y_{i}$, every artificial fish will explore the minimum target $Y_{\min }$ in the neighborhood partners. If $Y_{i}>Y_{\min }$, the fish will immediately move towards to the partner with $Y_{\min }$, while position $X_{i}$ changed into new position $X_{j}$. Each artificial fish will try its best to find the food concentration by changing its position and compare the food concentration (the minimum target) of the corresponding position with the bulletin board automatically. After a series of repeated optimization searching process, the optimal solution containing the best results will eventually be recorded in the bulletin board.

\subsubsection{Algorithm procedure}

The main procedure of transmission network planning model based on AFSA is as follows:

1)Input initial data, such as the artificial fish number, maximum number of iterations, value of visual, maximum number of prey attempts etc.;

2)Generate $N$ artificial fish individuals in feasible region using the random number generator to complete the artificial fish initialization. Then calculate and compare food concentration of each artificial fish at present position, record the minimum food concentration with corresponding position onto the bulletin board;

3)Respectively conduct prey and follow behavior, update the position of each artificial fish;

4)After updating of all artificial fish, compare the food concentration with the record on bulletin board. If the food concentration is larger than the bulletin board record, replace it;

5)Check whether the number of iterations reaches the maximum, output calculation results if it does; otherwise, go back to step 4. The algorithm procedure is shown as Figure 2.

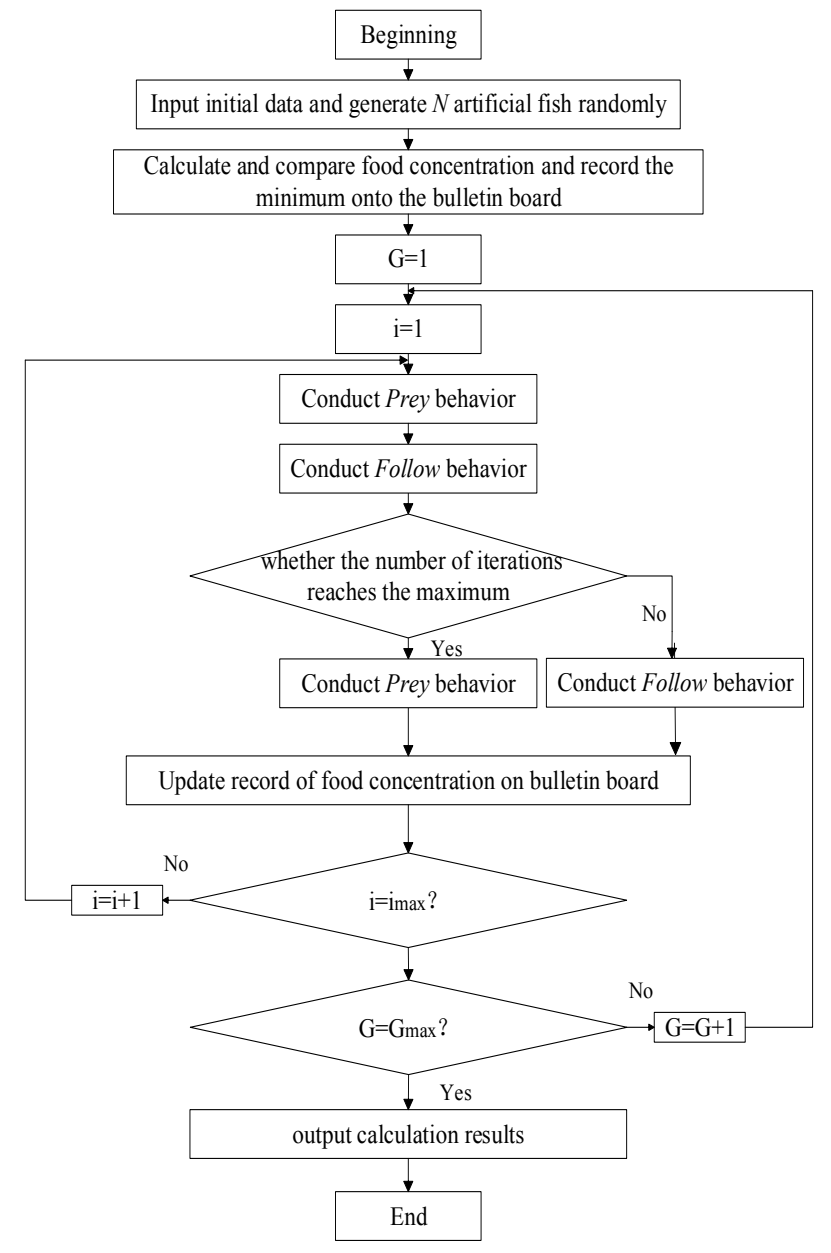

Fig.2 Main algorithm procedure of network planning based on improved AFSA

\section{EXAMPLE ANALYSIS}

In example analysis, the improved AFSA model with adaptive visual and step is used for solving the IEEE-6 Node transmission network planning problem. Main parameters of the algorithm are set as follows: artificial fish quantity $N=25$; maximum number of iterations $G_{\max }=100$; maximum prey attempts try $_{\max }=5$; maximum Visual $=10$; the improved adaptive visual and step can be calculated by Step $=\alpha \times$ Visual in the iteration process; congestion degree coefficient $\delta=0.3$. Programming the improved AFSA model based on MATLAB software, the optimal planning scheme of the minimum LCC is ultimately obtained with

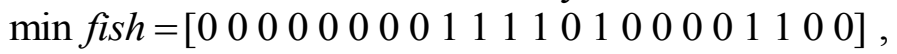
meaning the newly-built lines involved in the optimal scheme are line 9, 10,11,12,14, 19 and 20 and corresponding minimum LCC is $1.3259 \times 10^{9}$ yuan. The iteration process of AFSA model is shown in Figure 3, where the blue line represents the basic AFSA iteration process and the red line represents the improved AFSA iteration process. The corresponding optimal solution is shown in Figure 4. 


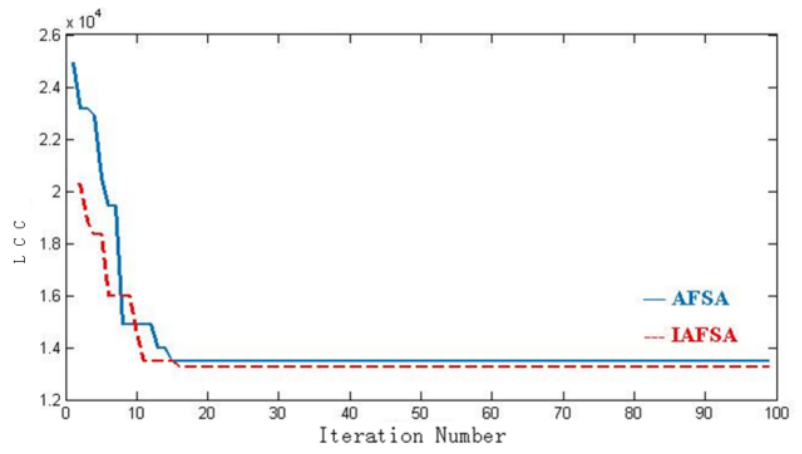

Fig.3 Improved AFSA iteration process

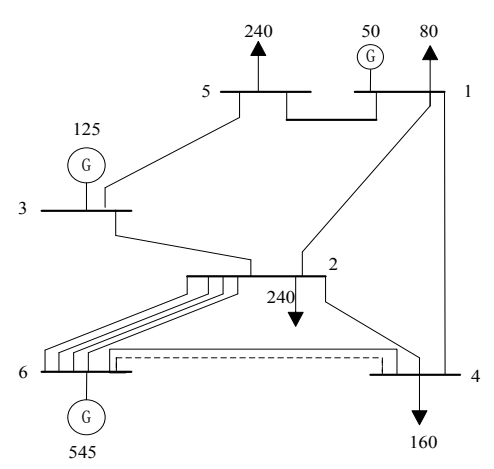

Fig.4 IEEE-6 Node transmission network planning results

Comparing the planning results of basic AFSA and improved AFSA, we can know that the minimum LCC of basic algorithm is $1.3483 \times 10^{9}$ yuan while improved algorithm is $1.3259 \times 10^{9}$ yuan, slightly lower than the former; the required time for 100 iterations of basic algorithm is 45.815 seconds while improved algorithm completes 100 iterations only in 22.833 seconds. As can be seen obviously, the planning result and iteration time of improved algorithm both precede basic algorithm.

\section{CONCLUSION}

This paper mainly researches on establishment and empirical study of transmission network planning model based on LCC theory and improved AFSA algorithm. The empirical results are ideal which verifies the applicability of the planning model proposed in this paper. In further study and practical application, the model can be used for solving complicated network planning and reducing the cost of network planning project to improve the economical efficiency.

\section{REFERENCES}

[1] Xue Hui. Study on the Application of Management Theory in the Distribution Network Engineering. North China Electric Power University, 2013

[2] Zhang Bingjie. Cost Management Research on the Electric Power Construction Project. North China Electric Power University, 2009

[3] Xu Lin. How to Strengthen LCC Management of Power Grid Construction Project. Financial Economy, 2011, 02:125-127.

[4] Lv Jianguo. Research on Electric Power Project Cost Management Based on LCC Theory. North China Electric Power University (Beijing), 2011

[5] Xu Tao. Research on Cost of Power Grid Planning Considering Economic and Reliability. South China University of Technology, 2011

[6] Liang Shuang, Fan Mingtian, Su Jian. Research on City Power Grid Performance Evaluation Framework, 2009, 05:8-11. 